\title{
Efficacy and Safety of Efavirenz in HIV Patients on Rifampin for Tuberculosis
}

Diana B. Pedral-Sampaio ${ }^{1,2}$, Carmosina R. Alves ${ }^{2}$, Eduardo M. Netto ${ }^{1,2}$, Carlos Brites ${ }^{1,2}$, Adriano S. Oliveira ${ }^{2}$ and Roberto Badaro ${ }^{1,2}$
Federal University of Bahia ${ }^{1}, C_{R E A I D S^{2}}$, Salvador, BA, Brazil

Forty-nine AIDS patients, most of who were antiretroviral therapy (ARV) naïve, with active tuberculosis, were treated with Rifampin $600 \mathrm{mg}$, Isoniazid 400mg and Pirazinamide $2 \mathrm{~g}$ daily. They also received ARV, consisting of Efavirenz $(600 \mathrm{mg} /$ day) plus 2 NRTIs. All patients were prospectively followed for at least 24 months. Baselines were: male/female ratio 2:1, mean age $34.7 \pm 9.4 \mathrm{yrs}$; weight $51 \pm 9.0 \mathrm{~kg}$, viral load $5.6 \pm 0.6 \mathrm{logs}, \mathrm{CD}_{4}$ cell count $101 \pm 128 \mathrm{cells} / \mathrm{mm}^{3}$. Follow up mean values of data logs of $\mathrm{VL}$ and $\mathrm{CD}_{4}{ }^{+}$cell $/ \mathrm{mm}^{3}$ counts were: $\mathrm{VL} 1.7$ and $\mathrm{CD}_{4}{ }^{+} 265 ; \mathrm{VL}$ 1.3 and $\mathrm{CD}_{4}{ }^{+} 251$; $\mathrm{VL} 1.4$ and $\mathrm{CD}_{4}{ }^{+} 326$ at 6,12 and 24 months, respectively. Weight gain changes were: $5 \pm 9.9 \pm 12$ and $21 \pm 16 \mathrm{~kg}$ respectively at 6,12 and 24 months. A non-concomitant ARV regimen was introduced at least three weeks after TB treatment initiation. Severe adverse reactions included rash (two), toxic hepatitis (six), Immune Reconstitution Syndrome (seven), and four deaths. We conclude that Efavirenz at a daily dose of $600 \mathrm{mg}$ is sufficient and safe to treat HIV/TB patients using a Rifampin containing regimen.

Key Words: Tuberculosis, HIV/AIDS, drug interaction, Rifampin, Efavirenz, antiretroviral therapy.

Eleven percent of all adult AIDS deaths are due to tuberculosis (TB) [1,2]. The goal of HIV and Mycobacterium tuberculosis co-infection management is to provide potent and safe antiretroviral therapy (ARV) and anti-tuberculosis treatment effective enough to cure and prevent recurrence and resistance $[3,4]$. However, increased frequency and severity of adverse reactions, including death, is well documented in TB-HIV co-infected patients during simultaneous treatment $[5,6]$. Several reports have documented the limitation of protease inhibitor (PI) containing regimens for the treatment of TB/AIDS patients due to drug-drug interaction between PIs and Rifampin (RMP) [7,8]. Alternatively, non-nucleoside reverse transcriptase inhibitors (NNRTIs) have been considered an adequate choice as an ARV regimen, Received on 17 March 2004; revised 27 May 2004.

Address for correspondence: Dr. Diana Sampaio. Hospital Universitário Professor Edgard Santos rua João das Botas S/ $\mathrm{n}^{\circ}$, Canela. Zip code: 40110-160 Salvador, Bahia, Brazil. Phone (5571) 2354901 Fax (5571) 2472756.E-mail: dpedral@ufba.br

The Brazilian Journal of Infectious Diseases 2004;8(3):211-216 (C) 2004 by The Brazilian Journal of Infectious Diseases and Contexto Publishing. All rights reserved. despite the induction of CYP450 by Rifampin. The available pharmacokinetic data provide evidence for only a $13 \%$ to $25 \%$ reduction in Efavirenz levels when coadministered with RMP [9], which is lower than Nevirapine (40\%), Delavaridine (90\%), and the $80 \%$ to 95\% reduction in pharmacokinetic levels that occur with PIs [7]. In spite of the low interaction of EFZ and RMP, the current recommendation is to increase the EFZ dose to $800 \mathrm{mg}$ per day when RMP is used. There is no data on the long-term clinical outcome of patients receiving either 600 or $800 \mathrm{mg} /$ day of Efavirenz simultaneously with Rifampin. Here we report a cohort of TB/AIDS patients, followed for 24 months, after being treated with an ARV regimen, containing $600 \mathrm{mg} /$ day Efavirenz and an antiTB treatment containing Rifampin.

\section{Materials and Methods}

\section{Design of the study}

An open label protocol designed to evaluate the safety and efficacy of simultaneous administration of 
standard tuberculosis treatment and an ARV regimen containing two NRTIs plus Efavirenz was conducted at the University Hospital of Salvador/Bahia-Brazil, from 1999 to 2002. Both local and national Institutional Review Boards approved the protocol and informed consent was obtained from all patients prior to enrollment. Initially, patients received either simultaneous treatment for TB and HIV or started ARV therapy within three weeks of commencing TB therapy. An interim analysis of 13 patients revealed a high rate of side effects. The protocol was therefore amended to initiate ARV at least three weeks after beginning TB therapy.

\section{Dose regimen and schedule}

TB treatment: For patients weighing more than $45 \mathrm{~kg}$, Isoniazide (400mg/daily) + Rifampin (600mg/ daily) was given for nine months and Pirazinamide ( $2 \mathrm{~g} /$ daily) for the first two months. Patients from 30$45 \mathrm{~kg}$ received Isoniazide $(300 \mathrm{mg} /$ daily) and Rifampin (450mg/daily) for nine months and Pirazinamide ( $1.5 \mathrm{~g} /$ daily) for the first two months. HAART: Two NRTIs at the recommended dose and Efavirenz $600 \mathrm{mg} /$ day (or $400 \mathrm{mg} /$ day for those weighing less than $40 \mathrm{~kg}$ ) was given initially with TB therapy, but then modified, due to adverse events, to start at least three weeks after beginning TB therapy.

\section{Patient population}

A total of 49 patients were enrolled in this study, according to the following inclusion/exclusion criteria:

\section{Inclusion criteria}

1. HIV infected patients with a viral load $=30,000$ copies/mL;

2. Clinical and radiological picture consistent with tuberculosis and/or one of the following: Positive AFB or TB culture or tissue biopsy suggestive of tuberculosis and

3. Ability to give informed consent.

\section{Exclusion criteria}

1. Patients with documented intolerance or resistance to Efavirenz;

2. Pregnant and breast-feeding women.

\section{Baseline and follow-up evaluation}

Clinical and demographic data were collected using a questionnaire and physical examination. Chest X-ray and laboratory profiles including WBC and ESR, biochemistry, electrolytes, AFB smear and TB culture were determined before treatment and twice a month during the first two months, then monthly until the ninth month, and every three months thereafter. $\mathrm{CD}_{4}^{+} / \mathrm{CD}_{8}^{+}$ cell counts and viral load measurement were performed at baseline and at months 1, 3, 6, 9, 12, 15, 18 and 24 after initiation of the combination treatment regimen. At each visit the patient was evaluated for the following: symptomatic toxic hepatitis (nausea, vomiting, jaundice), timing of onset of side effects, increased levels of hepatic enzymes, fever, dermatitis, neurological/ psychiatric abnormalities, lymph node enlargement, opportunistic infections and hospitalization. Signs and symptoms of tuberculosis were also checked at each patient visit.

\section{Results}

\section{Patient characteristics and clinical presentation}

The demographic characteristics and clinical status at baseline of the 49 patients are presented in Table 1. Mean age was 34.7 yrs (19-61 yrs). Thirty-four patients were male. The mean weight before treatment was $51.0 \pm 9.0 \mathrm{Kg}$, (range $35-71 \mathrm{Kg}$ ). Forty-one patients $(84 \%)$ were naïve for both treatments. Thirtyeight patients received both diagnoses simultaneously and three patients were previously diagnosed with HIV one to four years before their TB diagnosis, but were ARV naïve. Among the eight remaining patients, three were naïve for tuberculosis treatment but ARV experienced; another three patients were naïve for ARV 
treatment but had previous TB treatment. Two patients had previously received both TB and ARV treatment. The most frequent clinical presentations were pulmonary (49\%) and lymphadenopathy (24\%). Other clinical presentations included disseminated TB, lymphadenopathy and pulmonary involvement, peritoneal, pleural-pulmonary, pleural, and meningealencephalitis. Tuberculosis was microbiologically confirmed in $76 \%$ of cases while 12 patients were classified as presumptive TB cases.

\section{Response rate to $T B$ treatment}

Forty-nine patients participated in the trial. Three died during the first month of therapy. Four other patients stopped treatment during the first six months. Of these four patients, three were lost to follow-up. The fourth patient returned and was re-treated three times for TB but did not receive TB medications continuously for six months at any time of re-treatment. This patient died at month 24 with TB meningitis. Of the remaining 42 cured patients $(86 \%)$, two relapsed at month 12. Both were re-treated. One of them was cured and the other was lost to follow-up at month 15.

\section{$H I V$ virologic and immunologic responses to $A R V$}

The mean $\mathrm{CD}_{4}$ count at diagnosis was $101 \pm 128$ cells $/ \mathrm{mm}^{3}$ (median 44 , range $4-562$ cell $/ \mathrm{mm}^{3}$ ). The mean initial viral load (VL) was $5.6 \operatorname{logs} \pm 0.6$ (median: $5.7 \operatorname{logs}$ ranged 4.5-7.0 logs). The overall results of mean viral load evaluation at $3,6,12$, and 24 months after beginning therapy showed a significant decrease of $2.4,1.8,1.5$ and $1.3 \log \mathrm{s}^{10}$, respectively (p value < 0.001 for each time point). Also, a significant increase in mean $\mathrm{CD}_{4}^{+}$cell counts was observed in each time period $\left(204,265,251\right.$ and 326 cells $/ \mathrm{mm}^{3}$ at months 3 , 6, 12 and 24, respectively). Among 27 patients (55\%) who had adherence equal or greater than $90 \%$ to ARV treatment, the results were even more impressive. The mean $\mathrm{CD}_{4}^{+}$cell count increased from 92 cells $/ \mathrm{mm}^{3}$ at baseline to 262, 306, 298 and 431 at months 3, 6, 12 and 24of therapy, respectively. Also, mean VL decreased from $5.6 \operatorname{logs}$ at baseline to $1.7,0.8,0.3$ logs and undetectable levels at 3, 6, 12 and 24months, respectively (Figure 1). These results were significantly higher when compared with the non-compliant group, which had an initial $\mathrm{CD}_{4}^{+}$cell count of 112 cells $/ \mathrm{mm}^{3}$ and an increase to only 170, 221, 169 and 230 cells/ $\mathrm{mm}^{3}$ at months 3, 6, 12 and 24 , respectively $(\mathrm{p}<0.0001)$. Viral load results for the non-compliant group, which were $5.5 \mathrm{log}$ at baseline, also only decreased to $2.8,3.1,3.3$ and $2.5 \operatorname{logs}{ }^{10}$ at months 3 , $6,12$ and 24, respectively ( $\mathrm{p}=0.009)$. Comparison of $\mathrm{CD}_{4}^{+}$results between compliant and non-compliant patients was marginally significant ( $\mathrm{p}$ value $=0.056$, Figure 1). The main cause of non-compliance was adverse events that lead to temporarily stopping ARV, TB therapy or both regimens. Thirteen patients (27\%) initiated both treatments (TB and ARV) simultaneously or within an interval of less than three weeks. The remaining $(73.9 \%)$ initiated ARV drugs at least three weeks after TB therapy was begun. The mean time between initiation of TB and ARV therapy was $63 \pm$ 55 days (median 56 days).

\section{Adverse events}

Among the 13 patients initially enrolled in this protocol, three died very early in the course of therapy. The first patient, with a previous diagnosis of HIV, but ARV naive, developed renal failure during the first week and died at day 12 with acidosis and bleeding, despite dialysis. The $\mathrm{CD}_{4}{ }^{+}$count was 32 cells $/ \mathrm{mm}^{3}$. The second patient, also previously diagnosed with HIV, but ARV naïve, experienced sudden death, etiology unknown, at week four. This patient also had histoplasmosis, diagnosed by tongue biopsy, and $\mathrm{CD}_{4}^{+}$count of 6 cells $/ \mathrm{mm}^{3}$. The third patient, previously diagnosed with both HIV and TB, was diagnosed with histoplamosis on day 14 of TB therapy and died on day 21 . Among the initial 13 patients, we noted four cases of toxic hepatitis (31\%), and one case of drug reaction with eosinophilia and systemic symptoms. All these cases needed hospitalization due to side effects. Among the 36 patients who started ARV therapy after three weeks of TB therapy, there was one death, which occurred in a non-adherent patient who died of TB meningeal- 
encephalitis 24 months after the initiation of TB therapy. Among the group who initiated simultaneous therapies there were $4 / 13$ cases of toxic hepatitis (31\%, $\mathrm{p}<0.02)$ compared to the group that started ARV later who had 2/36 (5.6\%, $\mathrm{p}<0.02)$. Among the group who initiated ARV later, there was one case of rash and seven cases of neuropsychiatric side effects, such as insomnia, nightmare, and depression (mild to moderate) that did not lead to Efavirenz interruption during TB treatment. There were also seven cases of Immune Reconstitution Syndrome (IRS defined by appearance of $H$. zoster or reappearance of fever/ lymph node enlargement not related to TB recurrence); lymph node enlargement related to IRS occurred in six patients who originally presented with TB lymphadenopathy (Table 1).

\section{Discussion}

During the last decade we have treated more than $500 \mathrm{~TB} / \mathrm{HIV}$ patients at the infectious disease outpatient clinic at the Federal University Hospital of Bahia. Our trial, using a HAART regimen with Efavirenz in TB/HIV patients, documents a faster, more sustained and improved response in viral load, $\mathrm{CD}_{4}{ }^{+}$cell counts and clinical outcome to TB treatment, when compared to a regimen containing only two NRTIs.

Simultaneous administration of TB and ARV drug treatment for co-infected patients has been extensively reported $[3,8,10,11]$. Nevertheless, controversies still exist regarding the best regimens and appropriate schedule for the administration of both treatments. Rifampin, an essential drug component of TB treatment, is a potent inducer of cytochrome P-450$3 \mathrm{~A}$ in the intestinal wall and liver [12,13]. Protease inhibitor drug levels are critically affected by Rifampin [7]. Also, non-nucleoside reverse transcriptase inhibitors are affected by Rifampin. Efavirenz serum levels decrease approximately $15 \%$ to $25 \%$ in the presence of Rifampin $[9,12,13]$. The most recent guidelines for the management of co-infected HIV and TB patients recommend a HAART regimen using
Efavirenz at a higher dose (800mg daily), when simultaneously treating both infections [11]. But we found that TB and AIDS were controlled with Efavirenz at the regular dose of $600 \mathrm{mg} /$ day in the presence of Rifampin, regardless of the time of initiation of ARV therapy. However, by postponing ARV therapy for at least three weeks we documented a significant decrease in adverse reactions compared to the introduction of both regimens at the beginning of TB treatment. Although the number of patients receiving simultaneous treatment for $\mathrm{TB}$ and $\mathrm{HIV}$ was limited, the occurrence of serious adverse reactions, including toxic hepatitis and death, indicated that initiation of TB treatment without ARV drugs for at least three weeks was the best approach for the management of TB in our HIV co-infected patients.

The occurrence of immune reconstitution syndrome in seven patients (14\%) was higher when compared with a two NRTI regimen alone, and should be evaluated appropriately because of the possibility of misdiagnoses of TB relapse or other opportunistic infections [14-16].

Our study clearly demonstrates that increasing Efavirenz to $800 \mathrm{mg} /$ day when also using Rifampin is not necessary and may increase side effects. In addition, initiating tuberculosis treatment at least three to four weeks prior to HIV treatment resulted in a better outcome for these co-infected patients.

\section{Acknowledgements}

This work was supported by the Fundação Bahiana de Infectologia. The authors thank Beverly Putnam and Lisa Cabral for their careful revision of this manuscript.

\section{References}

1. Corbett E.L., Watt C.J., Walker N., et al. The growing burden of tuberculosis global trends and interaction with HIV epidemic. Arch Intern Med 2003;163:1009-21.

2. Collins K.R., Quinones-Mateu M.E., Toossi Z., Arts E.J. Impact of tuberculosis on HIV-replication, diversity, and disease progression. AIDS Rev 2002;4(3): 165-76. 
Table 1. Comparison between AIDS patient's who received simultaneous or postponed antiretroviral therapy during the treatment of tuberculosis

\begin{tabular}{|c|c|c|c|}
\hline Value of X +/- SD & Simultaneous*1 & Postponed & (p Value) \\
\hline \# Patients & 16 & 36 & \\
\hline Age (yrs) & $35.5+/-10.0$ & $34.4+/-9.3$ & \\
\hline Gender (ratio)3.3 & 3.0 & 2.0 & \\
\hline \multicolumn{4}{|l|}{ Viral load $(\log )$} \\
\hline Initial & $5.8+/-0.4$ & $5.5+/-0.7$ & \\
\hline 24 months & $2.0+/-2.4$ & $1.0+/-2.1$ & \\
\hline \multicolumn{4}{|l|}{$\mathrm{CD}_{4}$ counts $\left(\right.$ cells $\left./ \mathrm{mm}^{3}\right)$} \\
\hline Initial & $95+/-98$ & $103+/-140$ & \\
\hline 24 months & $337+/-226$ & $322+/-257$ & \\
\hline \multicolumn{4}{|l|}{ Clinical aspects } \\
\hline Pulmonary & $3(23.1 \%)$ & $21(58.3 \%)$ & $\mathrm{p}<0.03$ \\
\hline Extra pulmonary & $10(76.9 \%)$ & $15(41.7 \%)$ & \\
\hline \multicolumn{4}{|l|}{ Adverse events } \\
\hline Toxic hepatitis & $4(30.8 \%)$ & $2(5.6 \%)$ & $\mathrm{p}<0.02$ \\
\hline Rash/DRESS *2 & $1(7.7 \%)$ & $1(2.8 \%)$ & \\
\hline IRS*3 & $0(0 \%)$ & $7(19.4 \%)$ & \\
\hline Hospitalization & $11 / 13(84.6 \%)$ & $30 / 36(83.3 \%)$ & \\
\hline Neuropsychiatric effects & $0 / 13(0 \%)$ & $7 / 36(19.4 \%)$ & \\
\hline Death & $3(23.1 \%)$ & $1(2.8 \%)$ & $\mathrm{p}<0.02$ \\
\hline
\end{tabular}

*1 interval between TB and ARV therapy 0-3 weeks. *2 DRESS- drug rash with eosinophilia and systemic symptoms. *3 IRS-Immune Reconstitution Syndrome was defined as patient presenting with worsening TB signs, including reappearance of fever, lymph node enlargement or Herpes Zoster. 5/7 extra pulmonary TB.

Figure 1 - Trends in CD4 and Viral Load of Adherent and nonAdherent Patients with Tuberculosis and HIV

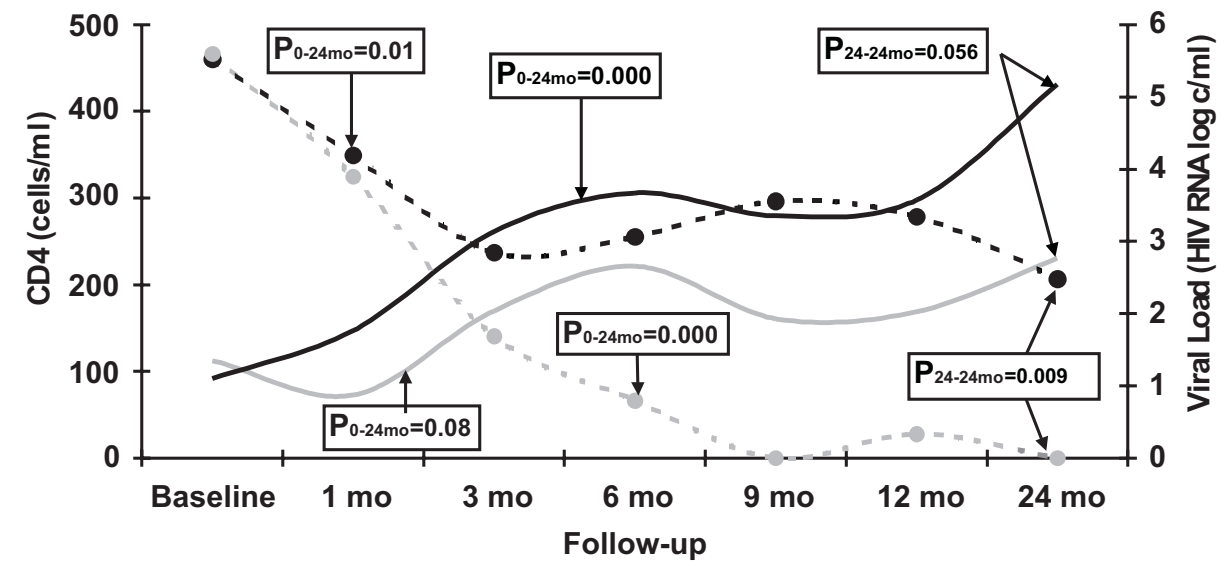

Legend

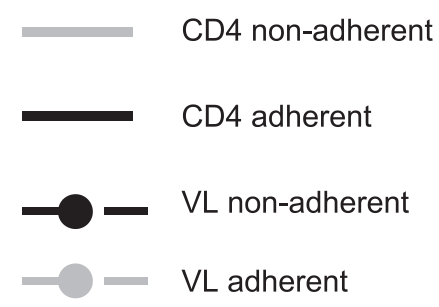

www.bjid.com.br 
3. Burman W.J., Jones B.E. Treatment of HIV-related Tuberculosis in the Era of Effective Antiretroviral Therapy. Am J Respir Crit Care Med 2001;164:7-12.

4. Munsiff S.S., Fugiwara P.I. Treatment of Tuberculosis in Patients Taking Antiretrovirals. The AIDS Reader 2000;10(2):102-8.

5. Pedral-Sampaio D.B., Netto E.M., Alcântara A.P., et al. Use of Standard Therapy for Tuberculosis is Associated with Increased Adverse Reactions in Patients with HIV. Braz J Infect Dis 1997; 1(3):123-30.

6. Harries A.D., Hargreaves N.J., Salaniponi F.M. Design of regimens for treating tuberculosis in patients with HIV infection, with particular reference to sub-Saharan Africa. Int J Tuberc Lung Dis 2001;5(12):1109-15.

7. U.S. Department of Health and Human Services. Updated guidelines for the use of rifabutin or Rifampin for the treatment and prevention of tuberculosis among HIVinfected patients taking protease inhibitors or nonnucleoside reverse transcriptase inhibitors. MMWR 2000;40:185-99.

8. Yew W.W. Clinically significant interactions with drugs used in the treatment of tuberculosis. Drug Saf 2002;25(2):111-33.

9. López-Cortés L.F., Rauiz-Valderas R., Viciana P., et al. Pharmacokinetic Interactions Between Efavirenz and Rifampicin in HIV-Infected Patients with Tuberculosis. Clin Pharmaco Kinet 2002;41(9):681-90.

10. Dean G.L., Edwards S.G., Ives N.J., et al. Treatment of tuberculosis in HIV-infected persons in the era of highly active antiretroviral therapy. AIDS 2002; 16:75-83 .

11. World Health Organization. Scaling Up. Antiretroviral Therapy in resource-limited settings. Guidelines for a Public Health Approach. Hammer S., Gibb D., Havlir D., et al [eds.]. Executive Summary, April 2002.

12. Finck C.K., Chrisman C.R., Baciewicz A.M., Self T.H. Rifampin and rifabutin drug interactions: an update. Arch Intern Med 2002;162(9):985-92.

13. Barret J.S., Joshi A.S., Chai M., et al. Population pharmacokinetic meta-analysis with efavirenz. Int J Clin Pharmacol Ther 2002;400(11):507-11.

14. Schluger N.W., Perez D., Liu Y.M. Reconstitution of immune responses to tuberculosis in patients with HIV infection who receive antiretroviral therapy. Chest 2002;122(2): 399-400.

15. Orlovic D., Smego R.A. Jr. Paradoxical tuberculosis reactions in HIV-infected patients. Int J Tuberc Lung Dis 2001;5(4): 370-5.

16. Domingo P., Torres O.H., Ris J., Vazquez G. Herpes zoster as an immune reconstitution disease after initiation of combination antiretroviral therapy in patients with human immunodeficiency virus type-1 infection. Am J Med 2001;110(8):662-3. 